\title{
Single-trial evaluative conditioning can be moderated by instructed forgetting
}

\author{
Anne Gast ${ }^{1}$ - Florian Kattner ${ }^{2}$
}

Published online: 19 February 2016

(C) Psychonomic Society, Inc. 2016

\begin{abstract}
Evaluative conditioning (EC) is a change in the valence of a conditioned stimulus (CS) due to previous pairing with an affective unconditioned stimulus (US). Several previous studies indicate that EC is related to memory of the CSUS pairs. Previous studies, however, typically cannot distinguish between the influence of CS-US knowledge during measurement and during encoding. In addition, by measuring rather than manipulating memory, they do not test the causal effect of memory on EC. The present study employed a "directed forgetting" procedure to the EC paradigm instructing participants to either forget or remember certain CS-US pairs. We found that EC effects after single learning trials were stronger for to-be-remembered than for to-beforgotten pairs. Manipulation checks showed that the forgetting manipulation also successfully modulated memory for the target pairs and reduced both retroactive and proactive interference on memory for other pairs. Item-based analyses further demonstrated that the size of EC depended on CS-US memory. The results suggest that EC relies on available memory during measurement of the EC effect.
\end{abstract}

Keywords Evaluative conditioning - Contingency memory * Contingency awareness $\cdot$ Directed forgetting $\cdot$ Proactive interference $\cdot$ Retroactive interference

Anne Gast and Florian Kattner contributed equally to this work.

Anne Gast

anne.gast@uni-koeln.de

Florian Kattner

kattner@wisc.edu

1 University of Cologne, Cologne, Germany

2 University of Wisconsin, Madison, WI, USA
Evaluative conditioning (EC) is the change of the valence of a stimulus that is due to the previous pairing with a positive or negative stimulus (De Houwer, 2007; Gast, Gawronski, \& Houwer, 2012). Although EC can, under some conditions, occur without memory for the pairings (Hütter \& Sweldens, 2013; Hütter, Sweldens, Stahl, Unkelbach, \& Klauer, 2012), memory for the pairings is a strong predictor of EC effects (e.g., Bar-Anan, De Houwer, \& Nosek, 2010; Hofmann, De Houwer, Perugini, Baeyens, \& Crombez, 2010; Pleyers, Corneille, Luminet, \& Yzerbyt, 2007; Stahl, Unkelbach, \& Corneille, 2009). A causal interpretation of the relation between memory and EC, however, is hampered by at least two ambiguities. First, most studies that demonstrate a relation between memory and EC did not experimentally manipulate but only measured it (Bar-Anan et al., 2010; Gawronski \& Walther, 2012; Hütter et al., 2012). Second, even if one would assume a causal influence of memory on EC, it is not clear whether this influence is exerted during the learning or during the measurement phase (e.g., Baeyens, Eelen, \& Van den Bergh, 1990; Gast, De Houwer, \& De Schryver, 2012; Gawronski \& Walther, 2012; Purkis \& Lipp, 2001). Typically, memory for the pairings is tested at the end of the experiment, shortly after or before the evaluative ratings. Any positive relation between EC and the memory measure may thus indicate an influence of memory that is present while the EC effect is measured ("recollection-during-measurement hypothesis"; see Gast et al., 2012a, b). However, as memory at this point is likely correlated with awareness of the stimulus pairings and their conscious encoding during the learning phase, the relation could also indicate an influence of conscious encoding of the pairings ("conscious-encoding hypothesis"; Gast et al., 2012a, b). In fact, several authors have investigated the relation between encoding and EC by manipulating attentional resources during the learning phase (and mostly found a positive relation; e.g., Blask, Walther, 
Halbeisen, \& Weil, 2012; Dedonder, Corneille, Bertinchamps, \& Yzerbyt, 2014; Field \& Moore, 2005; Fulcher \& Hammerl, 2001; Kattner, 2012; Pleyers, Corneille, Yzerbyt, \& Luminet, 2009; Walther, 2002).

The question of whether memory that is available at the measurement phase also has a genuine influence on EC has been neglected. In a recent study on this issue, the learning and measurement phases were separated by 9 or 10 days (Gast, De Houwer, et al., 2012). The authors found that EC was related to CS-US memory in the measurement session, but memory during the learning session had no additional predictive value. This result suggests a substantial influence of memory during the measurement on EC. Similar to previous findings, however, the interpretability of this result is limited by the fact that differences in memory were only measured but not manipulated. To more rigorously test the causal contribution of CS-US memory and to set it apart from encoding effects during the conditioning phase, it is necessary to experimentally manipulate memory after the learning phase and assess its influence on EC.

With the current study we wanted to do this by investigating whether EC is affected by directed-forgetting manipulations, which have previously been shown to reduce memory. In a typical directed-forgetting paradigm, participants study a list of items (e.g., words or pair associates) and are subsequently instructed to either forget or continue remembering a specified subset of the list. Many studies showed that participants recall less of the to-be-forgotten (F) items than of the to-beremembered (R) items ("remember-forget difference"; MacLeod, 1998). Moreover, more $\mathrm{R}$ items are typically recalled when the list also contains $\mathrm{F}$ items than when the list exclusively contains $\mathrm{R}$ items, a finding that is typically attributed to relief of interference from other $\mathrm{R}$ items ("directed-forgetting benefit"; e.g., Muther, 1965; Bjork, LaBerge, \& Legrand, 1968; for reviews, see Bjork, 1972; Johnson, 1994; MacLeod, 1998).

Directed forgetting is theoretically appealing because it demonstrates an intentional influence on memory that takes place after initial encoding. Furthermore, it does not require the studied CSs or USs to be presented again, thus avoiding additional exposure effects on the acquired stimulus evaluation. We therefore utilized an adapted version of the Bjork (1970) directed-forgetting paradigm to test whether EC effects are larger for pairs that had to be remembered than for pairs that had to be forgotten (i.e., a "remember-forget difference" on evaluative ratings).

Bjork's (1970) participants were asked to memorize several lists of nonword-word pairs. A change in background color (occurring in some lists) indicated that all previous items could be forgotten (i.e., F items; items after the change were $\mathrm{R}$ items). Memory for $\mathrm{R}$ items was tested immediately after each list, and some $\mathrm{F}$ words were tested "illegally" at the end of some final lists. Recall performance demonstrated both a remember-forget difference (better memory for $\mathrm{R}$ than for $\mathrm{F}$ items) and a directed-forgetting benefit in the form of a reduction of proactive interference due to $F$ items. That is, when told to forget the first items in a list, recall performance of subsequent $\mathrm{R}$ items did not depend on the number of items presented in the first part of the list.

To adapt this procedure to the EC paradigm, neutral nonwords (CSs) were paired with affective words (USs), and evaluative ratings of the CSs were included after presentation of each list. In addition, we changed the procedure so that both $\mathrm{F}$ and $\mathrm{R}$ items could occur at the beginning and at the end of the list. This avoids a confound with longer delays between encoding and test as well as with a context change (see Pastötter \& Bäuml, 2007; Sahakyan \& Kelley, 2002). We thus expanded the procedure by instructing participants to either forget none, the first, the second, or both halves (from here on denoted as "blocks") of a list of CSUS pairs. This allows testing directed-forgetting advantages in two ways: by analyzing differences in proactive interference (i.e., second-block recall as a function of first-block instruction) and retroactive interference (first-block recall as a function of second-block instruction). Because memory for $F$ words cannot be tested directly during the procedure-if participants noticed that we regularly tested $\mathrm{F}$ items this would probably make forget instructions ineffective - these analyses of directed-forgetting benefits during the main procedure are the most important manipulation checks. As additional manipulation checks, we used two measures from the end of the experiment: directed-forgetting benefits and remember-forget differences (memory difference between $\mathrm{R}$ and $\mathrm{F}$ items).

Our experiment was conducted to test the influence of remember versus forget instructions regarding specific past conditioning blocks on the size of the EC effects on conditioning pairs shown in these blocks (rememberforget difference on the EC effect). The EC effect is measured by evaluative ratings. As a manipulation check for the effectiveness of the remember versus forget instructions, we assessed memory effects of the remember versus forget instructions. Specifically, we did this (1) by assessing the memory benefit on stimuli that are presented closely to to-be-forgotten pairs (directed-forgetting benefit) during the main procedure, (2) by assessing the directed-forgetting benefit at the end of the whole procedure, and (3) by assessing the difference in memory for the to-be-remembered and the to-be-forgotten items at 
the end of the whole procedure (remember-forget difference on memory). ${ }^{1}$

\section{Method}

\section{Participants}

Twenty and thirty-two students from the University of Cologne volunteered to participate in two waves of our experiment. The second wave was originally designed as an exact replication of the first one but with slightly larger sample size. Because exactly the same method was used in both waves, we report the data collapsed across waves. ${ }^{2}$ Participants either received course credit or four Euros for compensation.

\section{Design}

The experiment had two experimental factors: the valence of the US with which a $\mathrm{CS}$ was paired (valence: $\mathrm{CS}_{\text {pos }}, \mathrm{CS}_{\text {neg }}$; within) and the type of instruction that the participant received about the specific conditioning block of which a CS pair was part (instruction: remember, forget; within).

\section{Material}

The stimuli used as CSs were 80 meaningless but pronounceable three-letter strings with medium association values from

\footnotetext{
${ }^{1}$ Prior to the experiment reported in this article, we conducted three additional experiments utilizing a different procedure to induce directed forgetting during EC. Specifically, each nonword-word (CS-US) pairing was presented repeatedly during the learning phase with different block structures being used in the three experiments. In Experiment $01(N=53)$, two $\mathrm{R}$ and two $\mathrm{F}$ blocks were presented (order counterbalanced), each consisting of four repetitions of four CS-US pairings; in Experiment 02 $(N=24)$, two R and two F blocks were presented (order counterbalanced) each consisting of eight repetitions of four CS-US pairings; and in Experiment $03(N=26)$, one $\mathrm{R}$ and one F block (order counterbalanced) each consisting of four repetitions of four CS-US pairings were presented. In all preexperiments, however, the instructions to forget particular pairings did not reduce CS-US memory (i.e., CS-cued recognition of the US) as compared to memory for the to-be-remembered CS-US pairings. Not surprisingly, directed forgetting did also not modulate the size of the EC effect. We therefore decided to apply a list-learning procedure that is more typical in directed forgetting research (and which deviates more from the standard EC paradigm), with (a) participants having to memorize a greater total number of pairings, (b) each pairing being presented only once, and (c) memory being tested both immediately after each list and at the end of the experiment via CS-cued recall (e.g., Bjork, 1970).

${ }^{2}$ Analyses including the factor wave yielded no significant main effects or interactions involving this factor except for a stronger remember-forget difference on the delayed measure in the first than in the second wave and better overall recall accuracy on second block items during the main procedure in the first compared to the second wave, which were both unexpected and difficult to explain. Analysing both waves separately showed that in each wave the crucial moderation of the EC effect was significant. Also at least one of the memory measures showed a significant directed-forgetting effect in each of the waves separately.
}

Archer (1960). We avoided stimuli that are close to German words. As USs we used 40 positive and 40 negative German two-syllable nouns that were translated from Moors et al. (2013) or taken from Schwibbe, Räder, Schwibbe, Borchardt, and Geiken-Pophanken (1994).

\section{Procedure}

After obtaining informed consent, the experimental program was started, and participants were given two response sheets (the second of which was covered at the beginning of the experiment) and instructions to memorize lists of nonwordword pairs.

Main procedure Each conditioning trial started with the presentation of the CS in the center of the screen. After $1 \mathrm{~s}$ the US appeared below the CS, and both stimuli remained on the screen for $5 \mathrm{~s}$. The next trial started after a 2-s blank interval.

The main phase of the experiment was structured as follows (see Table 1 for further illustration): Altogether 80 different CS-US pairs were presented, each in only one conditioning trial. The trials were divided into 20 blocks, each of which contained four CS-US pairing trials (two with positive and two with negative USs). The CS-US pairs within a block were presented in random order. After each block, participants either received the instruction to further remember $(\mathrm{R})$ or to forget (F) the pairs of the previous block. The instructions stated (in German), "Please keep remembering the pairs of the last yellow (light blue) block" after a remember block, and "You may now forget the pairs of the last yellow (light blue) block. These will not be tested," after a forget block.

After two consecutive blocks ("double blocks"), participants rated all eight previous CSs in random order according to how pleasant or unpleasant they sounded, by clicking on a scale ranging from -10 to +10 . If a double block contained at least one R block, immediate memory of two CS-US pairs from one $\mathrm{R}$ block (one $\mathrm{US}_{\text {pos }}$, one $\mathrm{US}_{\text {neg }}$ ) was then tested by presenting the CS (nonword) and asking the participant to write down on the provided response sheet which US (word) was paired with it.

The full design comprised four remember-remember (RR) double blocks, two remember-forget (RF) double blocks, two forget-remember (FR) double blocks, and two forget-forget (FF) double blocks (to allow testing proactive and retroactive interference of $F$ and $R$ items on $R$ items with equal power, there were twice as many RR blocks as other types of double blocks; see Table 1 for an illustration of the full block design). The double blocks were presented in random order. Thus, in total there were $12 \mathrm{R}$ blocks (with $48 \mathrm{R}$ pairs) and eight $\mathrm{F}$ blocks (with $32 \mathrm{~F}$ pairs). To help participants distinguish the two blocks of a double block, the first block of a double block was always presented against a light-blue background and the second against a yellow background, or 
Table 1 Specification of the block design

\begin{tabular}{|c|c|c|c|c|c|}
\hline Double block & Block & CS No. & Instruction & CSs in immediate memory test & CSs in final memory test \\
\hline \multirow[t]{2}{*}{1} & 1 & $1-4$ & $\mathrm{R}$ & 1,3 & 2,4 \\
\hline & 2 & $5-8$ & $\mathrm{R}$ & - & 6,8 \\
\hline \multirow[t]{2}{*}{2} & 3 & $9-12$ & $\mathrm{R}$ & - & 10,12 \\
\hline & 4 & $13-16$ & $\mathrm{R}$ & 13,15 & 14,16 \\
\hline \multirow[t]{2}{*}{3} & 5 & $17-20$ & $\mathrm{R}$ & 17,19 & 18,20 \\
\hline & 6 & $21-24$ & $\mathrm{~F}$ & - & 22,24 \\
\hline \multirow[t]{2}{*}{4} & 7 & $25-28$ & $\mathrm{~F}$ & - & 26,28 \\
\hline & 8 & $29-32$ & $\mathrm{R}$ & 29,31 & 30,32 \\
\hline \multirow[t]{2}{*}{5} & 9 & $33-36$ & $\mathrm{~F}$ & - & 34,36 \\
\hline & 10 & $37-40$ & $\mathrm{~F}$ & - & 38,40 \\
\hline \multirow[t]{2}{*}{6} & 11 & $41-44$ & $\mathrm{R}$ & 41,43 & 42,44 \\
\hline & 12 & $45-48$ & $\mathrm{R}$ & - & 46,48 \\
\hline \multirow[t]{2}{*}{7} & 13 & $49-52$ & $\mathrm{R}$ & - & 50,52 \\
\hline & 14 & $53-56$ & $\mathrm{R}$ & 53,55 & 54,56 \\
\hline \multirow[t]{2}{*}{8} & 15 & $57-60$ & $\mathrm{R}$ & 57,59 & 58,60 \\
\hline & 16 & $61-64$ & $\mathrm{~F}$ & - & 62,64 \\
\hline \multirow[t]{2}{*}{9} & 17 & $65-68$ & $\mathrm{~F}$ & - & 66,68 \\
\hline & 18 & $69-72$ & $\mathrm{R}$ & 69,71 & 70,72 \\
\hline \multirow[t]{2}{*}{10} & 19 & $73-76$ & $\mathrm{~F}$ & - & 74,76 \\
\hline & 20 & $77-80$ & $\mathrm{~F}$ & - & 78,80 \\
\hline
\end{tabular}

Note. The CS Nos. 1 and 2 refer to the $\mathrm{CS}_{\text {pos }}$ of a block, and the CS Nos. 3 and 4 refer to the $\mathrm{CS}_{\text {neg. }}$ of a block. The 10 double blocks as well as the four CS-US pairs within each block were presented in random order

vice versa, for half of the participants. The $\mathrm{R}$ or $\mathrm{F}$ instructions were always presented against a white background.

Additional measures After the last block, participants rated the valence of all $80 \mathrm{CSs}$ again in random order. Next, delayed CS-US memory of $\mathrm{F}$ and $\mathrm{R}$ blocks was tested in random order for two items (one positive and one negative) from each block (previously tested R items were not selected). Note that these "illegal" tests of $\mathrm{F}$ items (which are required for analyzing remember-forget differences) were only conducted at the end of the experiment in order to prevent interference with the instructions.

\section{Results}

\section{Directed forgetting effects on EC}

Figure 1a shows immediately measured EC scores (CSposCSneg) as a function of the different block types. A 2 (instruction) $\times 2$ (US valence) mixed-model ANOVA with instruction and US valence as within-subjects factors revealed a significant main effect of US valence, $F(1,51)=31.85, p<$ $.001, \eta_{\mathrm{G}}^{2}=0.16$, indicating an overall EC effect on immediately tested CS evaluations. More importantly for our research question, there was also a significant US valence $\times$ instruction interaction, $F(1,51)=14.38, p<.001, \eta^{2}{ }_{\mathrm{G}}=0.02$, demonstrating larger EC effects for R items $\left(M_{\text {pos }}=1.51, S D_{\text {pos }}=\right.$ 1.71 versus $\left.M_{\text {neg }}=-0.44, S D_{\text {neg }}=1.86\right)$ than for F items $\left(M_{\text {pos }}\right.$ $=0.95, S D_{\text {pos }}=1.64$ versus $\left.M_{\text {neg }}=0.03, S D_{\text {neg }}=1.45\right)$. There was no main effect of instruction, $F(1,51)=0.13, p=.724$.

Evaluative ratings of CSs at the end of the experiment (see Fig. 1b) also revealed a significant $\mathrm{EC}$ effect, $F(1,51)=22.62$, $p<.001, \eta_{\mathrm{G}}^{2}=0.07\left(M_{\text {pos }}=0.77, S D_{\text {pos }}=1.63\right.$ versus $M_{\text {neg }}=$ $\left.-0.17, S D_{\text {neg }}=1.74\right)$. However, there was no US valence $\times$ instruction interaction, $F(1,51)=0.16, p=.692$, indicating that remember/forget instructions did not have an impact on the delayed measure of EC (R blocks: $M_{\text {pos }}=0.78, S D_{\text {pos }}=$ $1.50, M_{\text {neg }}=-0.11, S D_{\text {neg }}=1.68 ; \mathrm{F}$ blocks: $M_{\text {pos }}=0.76, S D_{\text {pos }}$ $\left.=1.76, M_{\text {neg }}=-0.22, S D_{\text {neg }}=1.80\right)$. There was also no main effect of instruction, $F(1,51)=0.26, p=.615$.

\section{Manipulation checks: directed-forgetting effects on memory}

For testing whether our directed-forgetting manipulation was successful, we assessed (1) the directed-forgetting benefits measured immediately after each block, (2) the directedforgetting benefits measured at the end of the experiment, and (3) the remember-forget difference. All this measures were assessed on the ability to recall the correct US upon presentation of a CS. 


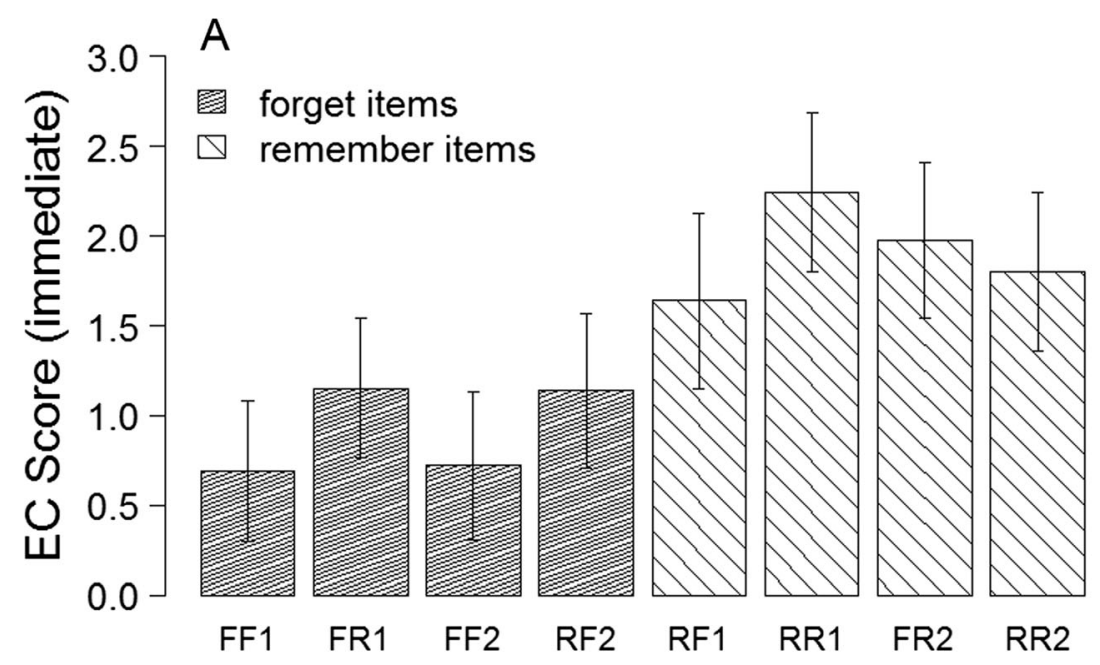

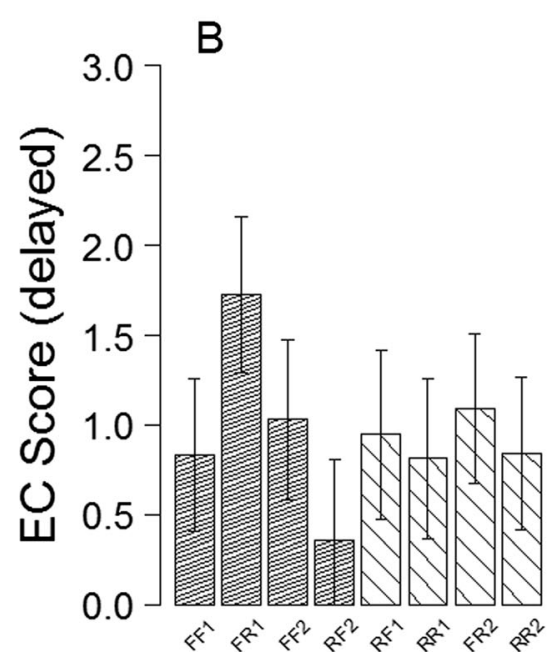

Fig. 1 EC effects and memory as a function of different types of double blocks, consisting of to-be-forgotten $(\mathrm{F})$ and to-be-remembered $(\mathrm{R})$ blocks, on different measures: Panel a shows immediate EC scores; Panel b shows CS-US recall performance at the end of the experiments; Panel $\mathbf{c}$ shows final EC scores. The $x$-axis shows the type of double block (FF: both to be forgotten, FR: first block to be forgotten, second block to

Directed-forgetting benefits on immediate memory Directed-forgetting benefits can in principle be tested with the immediate and the delayed memory tests, although the later test might yield less clear results due to multiple sources of inference potentially exerting an effect. Therefore, the directed-forgetting benefits on the immediate measure are our primary manipulation check. It was tested whether (a) F instructions in the second block reduced retroactive interference on $\mathrm{R}$ items of the first block and (b) $\mathrm{F}$ instructions in the first block reduced proactive interference on $\mathrm{R}$ items in the second block (compared to $\mathrm{R}$ instructions). Proactive and retroactive interference effects were thus evaluated by conducting two separate 2 (US valence: positive vs. negative) $\times 2$ (instruction: RR vs. RF/FR) repeated-measures ANOVAs

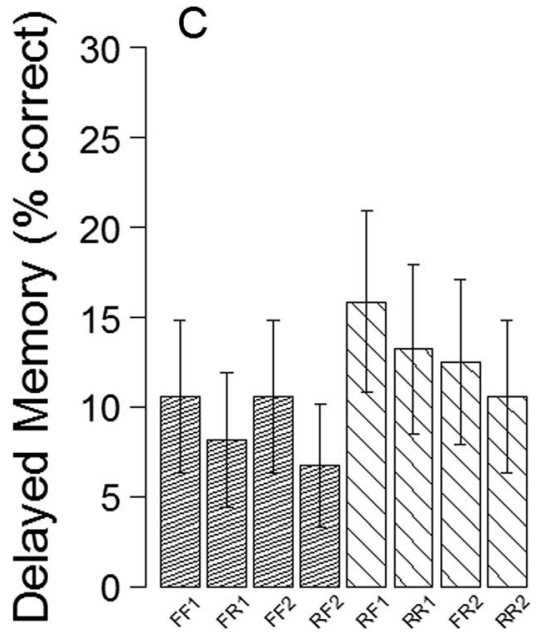

be remembered, RF: first block to be remembered, second block to be forgotten, RR: both blocks to be remembered). The numbers indicate whether the first (e.g., RR1) or second (e.g., RR2) block of the respective double block was analysed. Error bars represent standard errors of the mean

on recall accuracy of $\mathrm{R}$ items in the immediate tests. The effects of directed forgetting on immediate recall are illustrated in Fig. 2. We found a reduction of retroactive interference through instructed forgetting. That is, recall of to-beremembered CS-US pairs from a first block (in an RR or $\mathrm{RF}$ double block) was significantly better if participants were instructed to forget the second block of the double block (RF: $M=43.8 \%, S D=34.6 \%$ correct), than if they were instructed to remember the second block (RR: $M=34.1 \%$ correct, $S D=$ $34.3 \%), F(1,51)=4.44, p=.040, \eta^{2}{ }_{\mathrm{G}}=0.02$. There was also a significant main effect of the valence of the to-be recalled US (from a first block), $F(1,51)=4.08, p=.048, \eta^{2}{ }_{\mathrm{G}}=0.02$ $\left(M_{\text {pos }}=43.3 \%, S D_{\text {pos }}=32.7 \%, M_{\text {neg }}=34.6 \%, S D_{\text {neg }}=\right.$ $36.3 \%$ ), indicating better recall of pairs with positive USs. 


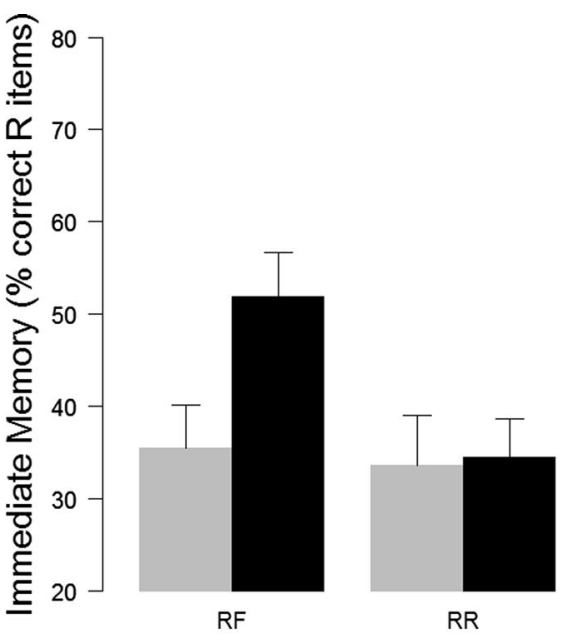

Retroactive Interference

Fig. 2 Immediate recall of CS-US pairings that were presented in the first (retroactive interference) or second (proactive interference) block of a double block, respectively. The nontested block of the same double block

There was no significant US valence $\times$ instruction (about the second block) interaction, $F(1,51)=3.53, p=.066$.

Instructed forgetting also reduced proactive interference: Immediate recall of $\mathrm{R}$ items from a second block was superior if the first block had to be forgotten (FR: $M=44.7 \%$ correct, $S D=36.8 \%$ ) than if it had to be remembered (RR: $M=$ $30.8 \%$ correct, $S D=36.5 \%), F(1,51)=7.87, p=.007$, $\eta_{\mathrm{G}}^{2}=0.04$. There was a also marginally significant main effect of US valence, $F(1,51)=3.64, p=.062, \eta_{\mathrm{G}}^{2}=0.01$, suggesting better recall of positive USs (from a second block; $M_{\text {pos }}=41.3 \%, S D_{\text {pos }}=38.5 \%, M_{\text {neg }}=34.1 \%, S D_{\text {neg }}=$ $35.7 \%$ ), but no US valence $\times$ instruction interaction, $F(1$, $51)=0.17, p=.684$.

Directed-forgetting benefits on delayed memory Directedforgetting benefits on delayed memory are a relatively unprecise measure because here the indirect nature of forgetting benefits on remember items is combined with the multiple sources of interference across the many phases of the experiment. We nevertheless report the results on this measure. Figure 1c depicts the recall accuracy in the recall test for the different double blocks (note that memory was tested for both $\mathrm{F}$ and $\mathrm{R}$ items in the delayed test). For the delayed memory test we found no release from retroactive interference by instructed forgetting, $F(1,51)=0.79, p=$ $.379\left(M_{\mathrm{RF}}=15.9 \%, S D_{\mathrm{RF}}=29.8 \%, M_{\mathrm{RR}}=13.2 \%\right.$, $\left.S D_{\mathrm{RR}}=17.8 \%\right)$. However, there was a main effect of US valence, $F(1,51)=4.90, p=.031, \eta_{\mathrm{G}}^{2}=0.02$, again with better memory for positive USs $(M=17.5 \%, S D=$ $27.0 \%)$ than for negative USs $(M=11.5 \%, S D=$ $21.5 \%)$ from the first blocks. There was no interaction between US valence and instruction, $F(1,51)=0.94, p=$ .338 .

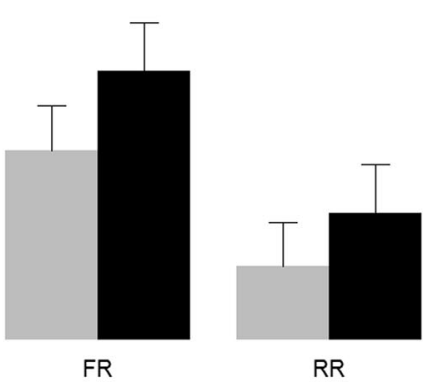

Proactive Interference

either had to be remembered (RR) or could be forgotten (RF, FR). Error bars represent standard errors of the mean

Directed forgetting did also not reduce proactive interference in the delayed memory measure, $F(1,51)=$ $0.76, p=.388\left(M_{\mathrm{FR}}=12.5 \%, S D_{\mathrm{FR}}=23.9 \%, M_{\mathrm{RR}}=\right.$ $10.6 \%, S D_{\mathrm{RR}}=20.0 \%$ ). There was also no main effect of US valence, $F(1,51)=1.84, p=.180$, and no US valence $\times$ instruction interaction, $F(1,51)=0.71, p=.403$.

Remember-forget difference Our third type of manipulation check consisted of assessing the difference in recall accuracy between $\mathrm{R}$ and $\mathrm{F}$ items at the end of the experiments. A 2 (instruction: F vs. R) $\times 2$ (block position: first vs. second block) repeated-measures ANOVA revealed that recall at the end of the experiment was significantly worse for $\mathrm{F}$ pairs than for R pairs, $F(1,51)=5.63, p=.022, \eta_{\mathrm{G}}^{2}=0.02\left(M_{\mathrm{F}}=9.0 \%\right.$, $S D_{\mathrm{F}}=20.2 \% ; M_{\mathrm{R}}=13.0 \%, S D_{\mathrm{R}}=23.3 \%$ ), while the position of the block (first vs. second block) had no significant main effect, $F(1,51)=1.63, p=.208\left(M_{1}=12.0 \%, S D_{1}=\right.$ $\left.22.7 \% ; M_{2}=10.1 \%, S D_{2}=20.8 \%\right)$. There was no instruction $\times$ position interaction, $F(1,51)=0.78, p=.381$.

\section{EC and CS-US memory}

In order to better understand the relation of memory and EC, we conducted multilevel analyses of the EC effect with CSUS memory as a factor at the level of individual pairs (see Gast et al., 2012a, b; Pleyers et al., 2007), based on linear mixed-effect models (using the R package lme4; R Core Team, 2014; Baayen, Davidson, \& Bates, 2008; Hoffman \& Rovine, 2007; Locker, Hoffman, \& Bovaird, 2007). Specifically, the effects of US valence, contingency memory $(0=$ not remembered $; 1=$ remembered $)$ and their interaction on the evaluative ratings were assessed while controlling for random effects at the level of participants and block (the 
random item factor was called "block" because we always tested one positive and one negative item from each block). Separate analyses were conducted based on immediate and delayed memory and valence measures. Note that because memory was tested for different items in the immediate and delayed test, these tests are based on different items. Because $\mathrm{F}$ items were never memory tested during the immediate memory test, this analysis only includes $\mathrm{R}$ items.

Immediate measures First of all, a model that specified US valence and contingency memory as fixed effects (including the interaction term) and participant and block as random effects (intercepts) was superior to a model that only contained US valence as a fixed factor (and the same random factor intercepts), $\chi^{2}(2)=18.03, p<.001$ (REML-based likelihood ratio test); note that a model with the interaction term significantly improved the fit compared to a model without the interaction term; $\chi^{2}(1)=14.84, p<.001$, AIC-corrected log likelihood ratio $=18.53$; the model without interaction term, however, did not significantly improve the fit compared to a model without contingency memory as a fixed factor, $\chi^{2}(1)=$ $3.19 ; p=.07$. Next, we found that the inclusion of byparticipant random slopes for US valence significantly improved the model, $\chi^{2}(2)=48.11, p<.001$, whereas byblock random slopes for US valence did not lead to any further improvements, $\chi^{2}(2)=0.43, p=.81$. Neither the addition of by-participant slopes for contingency memory, $\chi^{2}(1)=1.65, p$ $=.20$, nor by-block slopes for contingency memory improved the fit of the model, $\chi^{2}(2)=0.12, p=.94$. Table 2 summarizes the results for the fixed effects of the final model regarding the immediate measures (i.e. with participant and block as random factors and by-participant slopes for US valence). As can be seen, the model reveals significant main effects of US valence (the EC effect) as well as the crucial interaction indicating that CS-US memory increased the magnitude of immediate EC effects $\left(M_{\text {neg }, 0}=-0.31, S D_{\text {neg }, 0}=3.15 ; M_{\text {pos }, 0}=0.95, S D_{\text {pos, }}\right.$, ${ }_{0}=3.06 ; M_{\text {neg, } 1}=-0.66, S D_{\text {neg, } 1}=3.51 ; M_{\text {pos }, 1}=2.16, S D_{\text {pos }, 1}$ $=3.44$ ). The effect of contingency memory was only marginally significant, but remembered items, $M=0.90, S D=3.74$, tend to be evaluated more positively than for forgotten items, $M=0.28, S D=3.16$.

Delayed measures The same specification of an item-based linear mixed-effect model as used to model the immediate ratings (i.e., a model with random-factor intercepts for participant and block, random by-participant slopes for US valence, and fixed effects for US valence, contingency memory, and the interaction term) best described the relationship between contingency memory, US valence, and evaluative ratings of those items that were tested at the end of the experiment, $\chi^{2}(2)$ $=25.25, p<.001$ (relative to an otherwise identical model that did not contain the interaction term). The results of the fixed effects on delayed evaluative ratings are described in Table 3.
Table 2 Fixed effects of the linear mixed-effects model of immediate evaluative ratings (using REML estimation)

\begin{tabular}{llll}
\hline Predictor & Estimate $(S E)$ & $t$ & $p$ \\
\hline Intercept & $0.34(0.22)$ & 1.58 & .12 \\
US valence & $0.69(0.20)$ & 3.54 & $<.001$ \\
Contingency memory & $0.38(0.22)$ & 1.75 & .08 \\
US valence $\times$ contingency memory & $0.62(0.22)$ & 2.85 & .004 \\
\hline
\end{tabular}

Again, the analysis revealed a significant EC effect, a main effect of contingency memory (with more positive evaluations of remembered items, $M=1.49, S D=3.65$, than for forgotten items, $M=0.16, S D=3.11$ ), as well as an interaction indicating that the EC effect was larger for CS-US pairs that were correctly recalled at the end of the experiment $\left(M_{\text {neg, }, 0}=-0.17\right.$; $M_{\mathrm{pos}, 0}=0.50 ; M_{\text {neg, } 1}=-0.01 ; M_{\mathrm{pos}, 1}=2.60 ; S D$ s between 2.97 and 3.95).

\section{Discussion}

Participants were presented with a series of blocks, each containing several single CS-US presentations. After each block, participants were either instructed to continue to remember or forget the pairings from the block. Besides an effect on memory, we found that these instructions influenced CS evaluations as measured immediately after each double block: EC effects were stronger for to-be-remembered CS-US pairs than for to-be-forgotten pairs.

Importantly, we have different pieces of evidence that our directed forgetting manipulation actually influenced CS-US memory. First, a delayed recall test at the end of the experiments showed that $\mathrm{R}$ pairs were more likely to be recalled than $F$ pairs (remember-forget difference). Second, immediate recall showed directed-forgetting benefits, with memory for $\mathrm{R}$ items from a second block being superior when $\mathrm{F}$ items were presented in the previous block (reduction of proactive interference). Likewise, memory for $\mathrm{R}$ items from a first block was superior when they were followed by $\mathrm{F}$ items (reduction of retroactive interference). The delayed memory measure did not reveal effects on proactive or retroactive interference. This, however, was to be expected in the present design with

Table 3 Fixed effects of the linear mixed-effects model of the delayed evaluative ratings (using REML estimation)

\begin{tabular}{llll}
\hline Predictor & Estimate $(S E)$ & $t$ & $p$ \\
\hline Intercept & $0.18(0.19)$ & 0.94 & .35 \\
US valence & $0.34(0.10)$ & 3.58 & $<.001$ \\
Contingency memory & $1.02(0.21)$ & 4.87 & $<.001$ \\
US valence $\times$ contingency memory & $0.93(0.21)$ & 4.52 & $<.001$ \\
\hline
\end{tabular}


several $\mathrm{R}$ and $\mathrm{F}$ blocks being presented successively. It is likely that proactive and retroactive interference does not only occur within the defined double blocks but also between adjacent double blocks. Hence, interference processes from within a double block may have been overwritten by the multiple sources of interference from across the entire procedure.

An important feature of our study is that we did not only (like most previous studies) measure but also manipulated memory. This strongly suggests that the observed difference in EC between to-be-remembered and to-be-forgotten CS-US pairs is due to a causal influence of memory on stimulus evaluations. The findings support the hypothesis that the relation between EC and contingency awareness is not only due to differences in encoding during conditioning but also subject to later influences on CS-US memory. From a process perspective, it is important to point out that encoding is not necessarily finished with the end of the conditioning phase but might continue afterwards with rehearsal or consolidation processes. It is a complicated question that goes beyond the scope of this paper whether the forgetting instruction had an influence on an existing memory trace or interrupted ongoing encoding. Either way, the results suggest that, in line with the "recollection during measurement" hypothesis (Gast, De Houwer, et al., 2012), it is crucial for evaluative learning what can be remembered during measurement of the EC effect. Importantly, the results of an item-based analysis of the impact of contiguity memory on EC are compatible with the abovementioned results by showing that EC effects were larger for CSs for which the associated US could be recalled. This was the case both if the EC effect and recall were measured immediately after each block and at the end of the experiment.

While directed forgetting moderated the immediately measured EC effect, we did not find such an effect on the delayed measure. We think that the absence of this effect is not very surprising because directed forgetting is supposed to exert a more reliable and direct influence on immediate memory, whereas memory at the end of the experiment may be subject to decay and unsystematic interfering information. In fact, the finding that memory manipulations influence later EC effects less reliably fits well with the recollection-during-measurement hypothesis (Gast, De Houwer, et al., 2012), assuming memory at the time when the EC effect is measured to be a better predictor of EC than earlier memory. An alternative interpretation of the absence of a moderation of delayed EC is that EC effects measured with a delay might be based on more consolidated memory traces and thus be less susceptible to influences of intentional forgetting. More research is needed to gain a clearer view on the differential susceptibility of delayed and immediately measured EC effects to memory manipulations.

A limitation to consider when interpreting the results of the present study is that we only found the reported pattern of results with the described experimental paradigm that is very similar to the list-learning procedure by Bjork (1970) with single presentations. As mentioned, we ran some previous studies with repeated presentations that did not show an effect of the instructions - neither on the memory measures nor on the EC effect. These null findings thus suggest the ineffectiveness of the used instructed-forgetting manipulations to change memory that is based on repeated presentations. It does not allow more specific conclusion on EC. We thus currently cannot tell whether EC that is based on repeated presentations could be changed with a forgetting instruction that is strong enough to also change declarative memory for repeated pairings.

Another limitation of our study is that we only measured EC effects with explicit ratings. The main reason for using only a rating was that the previously mentioned studies suggested that we needed a design with several blocks, similar to the studies by Bjork (1970) and other directed-forgetting studies. The inclusion of an implicit measure after each double block of such a design would not only make the task exceedingly long, but the increasing experience on the implicit task would also make the results from it difficult to interpret.

Nevertheless, we want to acknowledge the possibility that the reported pattern of results would not have been found on an implicit measure of CS evaluations. There would be different explanations for such an outcome. First, implicit measures are often less reliable than explicit ratings, which can account for differences in the result pattern. Second, it is often argued that explicit and implicit measures pick up on different underlying processes of the EC effect to different degrees. It is possible that a procedure like the one used in the current experiment leads to EC effects that are mainly based on propositional processes and that specifically such propositional processes can be changed by remember-forget instructions. Third, it is conceivable that the reported pattern of results may be partly due to demand characteristics. Participants may have thought they were supposed to base the evaluative ratings of the CSs on the valence of the USs - and more so for those pairs that they were supposed to remember. Regarding this possibility, please note, however, that we explicitly asked participants to base their ratings on the sound of the nonwords. It is therefore unlikely that participants thought that a memory-based rating was the task. Please also note that all these explanations are only given for the possibility that the result pattern might not be found on an implicit measure, which we did not test due to previously described technical difficulties. Currently, we can only point to the interpretational limitations due to only using explicit rating measures.

To sum up, the present study shows that manipulations of memory through directed forgetting instructions influence the magnitude of EC. Stronger EC effects were found when participants were instructed to remember the respective CS-US pair after its presentation than when they were instructed to 
forget the pair. Moreover, the remember-forget instructions exerted the expected influence on CS-US memory. That is, we found (a) a recall difference between to-be-remembered and to-be-forgotten items on the delayed memory measure and (b) a reduction of proactive and retroactive interference through forgetting instructions on the immediate memory measure. Item-based analyses further showed that both immediate and delayed EC effects depended on whether participants were able to actually recall the US that was paired with the respective CS in the immediate and delayed memory tests, respectively. Taken together, these findings provide convincing evidence for the assumption that EC is at least partly based on the available memory at the time of testing (Gast et al., 2012a). These findings are of crucial importance for understanding the mental representations underlying EC. While many researchers agree that resources for conscious encoding increase EC effects, there is little consensus on whether the conscious knowledge that has been acquired in this resourcedependent process is needed when the EC effect is actually expressed. The current results suggest that conscious memory of the CS-US pairings that can be retrieved is an important factor.

Author Note The research reported in this paper was supported by grant GA 1520/2-1 from the Deutsche Forschungsgemeinschaft awarded to Anne Gast.

\section{References}

Archer, E. J. (1960). Re-evaluation of the meaningfulness of all possible CVC trigrams. Psychological Monographs: General and Applied, 74(10), 1-23. doi:10.1037/h0093758

Baayen, R. H., Davidson, D. J., \& Bates, D. M. (2008). Mixedeffects modeling with crossed random effects for subjects and items. Journal of Memory and Language, 59, 390-412. doi: 10.1016/j.jml.2007.12.005

Baeyens, F., Eelen, P., \& Van den Bergh, O. (1990). Contingency awareness in evaluative conditioning: A case for unaware affective-evaluative learning. Cognition \& Emotion, 4, 3-18.

Bar-Anan, Y., De Houwer, J., \& Nosek, B. A. (2010). Evaluative conditioning and conscious knowledge of contingencies: A correlational investigation with large samples. Quarterly Journal of Experimental Psychology, 63, 2313-2335. doi:10. 1080/17470211003802442

Bjork, R. A. (1970). Positive forgetting: The noninterference of items intentionally forgotten. Journal of Verbal Learning and Verbal Behavior, 9, 225-268.

Bjork, R. A. (1972). Theoretical implications of directed forgetting. In A. W. Melton \& E. Martin (Eds.), Coding processes in human memory (pp. 217-235). Washington: Winston.

Bjork, R. A., LaBerge, D., \& Legrand, R. (1968). The modification of short-term memory through instructions to forget. Psychonomic Science, 10, 55-56.

Blask, K., Walther, E., Halbeisen, G., \& Weil, R. (2012). At the crossroads: Attention, contingency awareness, and evaluative conditioning. Learning and Motivation, 43, 99-106. doi:10.1016/j.lmot.2012. 03.004
R Core Team (2014). R: A language and environment for statistical computing [Computer software]. Vienna: R Foundation for Statistical Computing. Retrieved from http://www.r-project.org

De Houwer, J. (2007). A conceptual and theoretical analysis of evaluative conditioning. The Spanish Journal of Psychology, 10, 230-241.

Dedonder, J., Corneille, O., Bertinchamps, D., \& Yzerbyt, V. (2014). Overcoming correlational pitfalls: Experimental evidence suggests that evaluative conditioning occurs for explicit but not implicit encoding of CS-US pairings. Social Psychological and Personality Science, 5, 250-257. doi:10.1177/1948550613490969

Field, A. P., \& Moore, A. C. (2005). Dissociating the effects of attention and contingency awareness on evaluative conditioning effects in the visual paradigm. Cognition and Emotion, 19, 217-243. doi:10. 1080/02699930441000292

Fulcher, E. P., \& Hammerl, M. (2001). When all is revealed: A dissociation between evaluative learning and contingency awareness. Consciousness and Cognition, 10, 524-549. doi: 10.1006/cog.2001.0525

Gast, A., De Houwer, J., \& De Schryver, M. (2012a). Evaluative conditioning can be modulated by memory of the CS-US pairings at the time of testing. Learning and Motivation, 43, 116-126.

Gast, A., Gawronski, B., \& De Houwer, J. (2012b). Evaluative conditioning: Recent developments and future directions. Learning and Motivation, 43, 79-88.

Gawronski, B., \& Walther, E. (2012). What do memory data tell us about the role of contingency awareness in evaluative conditioning? Journal of Experimental Social Psychology, 48, 617-623. doi:10. 1016/j.jesp.2012.01.002

Hoffman, L., \& Rovine, M. J. (2007). Multilevel models for the experimental psychologist: Foundations and illustrative examples. Behavior Research Methods, 39, 101-117. doi:10.3758/ bf03192848

Hofmann, W., De Houwer, J., Perugini, M., Baeyens, F., \& Crombez, G. (2010). Evaluative conditioning in humans: A meta-analysis. Psychological Bulletin, 136, 390-421. doi:10. 1037/a0018916

Hütter, M., \& Sweldens, S. (2013). Implicit misattribution of evaluative responses: Contingency-unaware evaluative conditioning requires simultaneous stimulus presentations. Journal of Experimental Psychology: General, 142, 638-643. doi:10.1037/a0029989

Hütter, M., Sweldens, S., Stahl, C., Unkelbach, C., \& Klauer, K. C. (2012). Dissociating contingency awareness and conditioned attitudes: Evidence of contingency-unaware evaluative conditioning. Journal of Experimental Psychology: General, 141, 539-557. doi: 10.1037/a0026477

Johnson, H. M. (1994). Processes of successful intentional forgetting. Psychological Bulletin, 116, 274-292.

Kattner, F. (2012). Revisiting the relation between contingency awareness and attention: Evaluative conditioning relies on a contingency focus. Cognition and Emotion, 26, 166-175. doi:10.1080/02699931.2011. 565036

Locker, L., Hoffman, L., \& Bovaird, J. A. (2007). On the use of multilevel modeling as an alternative to items analysis in psycholinguistic research. Behavior Research Methods, 39, 723-730. doi:10.3758/BF03192962

MacLeod, C. M. (1998). Directed forgetting. In J. M. Golding \& C. M. MacLeod (Eds.), Intentional forgetting: Interdisciplinary approaches (pp. 1-57). Mahwah: Erlbaum.

Moors, A., De Houwer, J., Hermans, D., Wanmaker, S., van Schie, K., Van Harmelen, A., ... Brysbaert, M. (2013). Norms of valence, arousal, dominance, and age of acquisition for 4300 Dutch words. Behavior Research Methods, 45, 169-177. doi:10.3758/s13428012-0243-8

Muther, W. S. (1965). Erasure or portioning in short-term memory. Psychonomic Science, 3, 429-430. 
Pastötter, B., \& Bäuml, K.-H. (2007). The crucial role of postcue encoding in directed forgetting and context-dependent forgetting. Journal of Experimental Psychology: Learning, Memory, and Cognition, 33, 977-982.

Pleyers, G., Corneille, O., Luminet, O., \& Yzerbyt, V. (2007). Aware and (dis)liking: Item-based analyses reveal that valence acquisition via evaluative conditioning emerges only when there is contingency awareness. Journal of Experimental Psychology: Learning, Memory, and Cognition, 33, 130-144. doi:10.1037/0278-7393.33. 1.130

Pleyers, G., Corneille, O., Yzerbyt, V., \& Luminet, O. (2009). Evaluative conditioning may incur attentional costs. Journal of Experimental Psychology: Animal Behavior Processes, 35, 279-285. doi:10.1037/ a0013429

Purkis, H. M., \& Lipp, O. V. (2001). Does affective learning exist in the absence of contingency awareness? Learning and Motivation, 32, 84-99.
Sahakyan, L., \& Kelley, C. M. (2002). A contextual change account of the directed forgetting effect. Journal of Experimental Psychology: Learning, Memory, and Cognition, 28, 1064-1072.

Schwibbe, M., Räder, K., Schwibbe, G., Borchardt, M., \& Geiken-Pophanken, G. (1994). Zum emotionalen Gehalt von Substantiven, Adjektiven und Verben. In W. Hager \& M. Hasselhorn (Eds.), Handbuch deutschsprachiger Wortnormen (pp. 272-284). Göttingen: Hogrefe.

Stahl, C., Unkelbach, C., \& Corneille, O. (2009). On the respective contributions of awareness of unconditioned stimulus valence and unconditioned stimulus identity in attitude formation through evaluative conditioning. Journal of Personality and Social Psychology, 97, 404-420. doi:10.1037/a0016196

Walther, E. (2002). Guilty by mere association: Evaluative conditioning and the spreading attitude effect. Journal of Personality and Social Psychology, 82, 919-934. doi:10.1037/0022-3514.82.6.919 